Service social

\title{
Les travailleurs de rue, témoins-acteurs dans les zones grises de la lutte contre l'intimidation
}

\section{Annie Fontaine}

Volume 62, numéro 1, 2016

Ensemble contre l'intimidation : une responsabilité partagée

URI : https://id.erudit.org/iderudit/1036335ar

DOI : https://doi.org/10.7202/1036335ar

Aller au sommaire du numéro

\section{Éditeur(s)}

École de service social de l’Université Laval

ISSN

1708-1734 (numérique)

Découvrir la revue

Citer cet article

Fontaine, A. (2016). Les travailleurs de rue, témoins-acteurs dans les zones grises de la lutte contre l'intimidation. Service social, 62(1), 57-71. https://doi.org/10.7202/1036335ar

\section{Résumé de l'article}

Faisant suite à un mémoire déposé dans le cadre du Forum de lutte contre l'intimidation organisé en 2014 par le gouvernement du Québec, cet article met en lumière comment la position de proximité des travailleurs de rue dans les milieux de vie des jeunes leur permet d'adopter une lecture globale des dynamiques d'intimidation et des conditions sociales dans lesquelles émerge ce phénomène. Cet article témoigne également de la contribution potentielle de ces intervenants hors murs que sont les travailleurs de rue dans la prévention de l'intimidation et dans l'intervention auprès de ceux qui sont impliqués. Enfin, cet article fait ressortir quelques conditions à préserver pour permettre aux travailleurs de rue de jouer pleinement leur rôle dans les zones grises de la lutte contre l’intimidation. 


\title{
Les travailleurs de rue, témoins-acteurs dans les zones grises de la lutte contre l'intimidation
}

\author{
FONTAINE, Annie \\ Professeure adjointe \\ École de service social, Université Laval
}

\section{RESUMÉ}

Faisant suite à un mémoire déposé dans le cadre du Forum de lutte contre l'intimidation organisé en 2014 par le gouvernement du Québec, cet article met en lumière comment la position de proximité des travailleurs de rue dans les milieux de vie des jeunes leur permet d'adopter une lecture globale des dynamiques d'intimidation et des conditions sociales dans lesquelles émerge ce phénomène. Cet article témoigne également de la contribution potentielle de ces intervenants hors murs que sont les travailleurs de rue dans la prévention de l'intimidation et dans l'intervention auprès de ceux qui sont impliqués. Enfin, cet article fait ressortir quelques conditions à préserver pour permettre aux travailleurs de rue de jouer pleinement leur rôle dans les zones grises de la lutte contre l'intimidation.

Mots-clés : intimidation, délinquance, déterminants sociaux de la santé, oppression, liens sociaux, travail de rue, action communautaire, approche globale, proximité, ruptures sociales, milieux de vie.

\begin{abstract}
Following a report produced for the Forum de lutte contre l'intimidation organized in 2014 by the government of Quebec, this article reports the point of view that adopt the social streetworkers on the phenomenon of intimidation from the position of closeness which allows their presence in the daylife environment. It presents the global reading which makes these outsider social workers of the dynamics of the intimidation and of the social conditions within which emerges that phenomenon. It also testifies of the potential contribution of streetworkers in the prevention of the intimidation and in the intervention with those who are concerned. Finally, this article highligths some conditions to be protected to allow the streetworkers to play completely their role in the grey area of the fight against intimidation.
\end{abstract}

Keys word : bullying, delinquency, social determinants of health, oppression, social links, social streetwork, community action, global approach, closeness, social disruptions, living environment. 


\section{INTRODUCTION}

Prenant diverses figures de relations de pouvoir interpersonnelles ou collectives, l'intimidation met en scène plusieurs enjeux auxquels sont confrontées les personnes qui vivent une ou plusieurs formes de rupture au sein de la configuration de liens sociaux qui les attachent à la société (Paugam, 2014). Inscrite dans un engrenage au sein duquel sont difficiles à distinguer les causes et les conséquences de cette forme de violence, l'intimidation gagne à être considérée sous l'angle des causes structurelles qui l'animent et de son inscription dans une trajectoire souvent marquée par diverses ruptures sociales (avec la famille, les pairs, l'école, le travail, la loi, etc.).

L'approche globale et généraliste du travail de rue peut être mise à profit de manière directe et indirecte pour contrer ce phénomène en tissant une passerelle avec les individus et les groupes dont les liens avec les espaces de socialisation et d'intégration sociale sont rompus ou fragilisés (Bondu, 1998; Cheval, 2001; Fontaine, 2003; Fontaine, 2011). La position de proximité des travailleurs de rue dans les milieux de vie des jeunes les place dans une position de témoins de la complexité des dynamiques qu'ils vivent et aiguille ces acteurs pour moduler une intervention ajustée aux besoins de ceux qu'ils accompagnent (Clément, Gélineau et McKay, 2009; Fontaine, 2011).

Comme d'autres auteurs (Bibeau et Perreault, 1995; Tétreault et Girard, 2007; Martel, 2008; Carle, 2014) ont mis en lumière le potentiel de ce mode d'intervention généraliste pour agir sur divers problèmes sociaux spécifiques (toxicomanie et prostitution, gangs de rue, itinérance), le présent article sous-tend l'hypothèse que c'est justement le caractère global de cette pratique de proximité qui en assure l'adéquation (Clément, Gélineau et McKay, 2009; Lamoureux et al., 2009; Fontaine, 2013a). Inspiré d'un mémoire rédigé en collaboration avec Steve Richard, représentant du Regroupement des organismes communautaires québécois pour le travail de rue (ROCQTR) au Forum de lutte contre l'intimidation (Fontaine et Richard, 2014), cet article explore la manière dont la présence des travailleurs de rue dans l'univers des jeunes peut être mobilisée pour prévenir et contrer l'intimidation. Après avoir illustré la propension des travailleurs de rue à considérer les conditions et les trajectoires à l'intérieur desquelles émergent des problèmes comme l'intimidation, il décrit comment l'immersion de ces intervenants dans les milieux de vie peut favoriser l'appropriation du pouvoir d'agir des jeunes sur leurs réalités. Éclairant quelques conditions nécessaires à mettre en place pour soutenir le déploiement efficace d'une telle stratégie d'action, ce texte conclut sur la nécessaire marge de manœuvre dont doivent disposer les travailleurs de rue pour jouer un rôle significatif auprès des jeunes, de leur entourage et plus largement dans la communauté. 


\section{L'intimidation, un problème social à envisager à la lumière d'une lecture globale des conditions d'existence des jeunes}

La présence des travailleurs de rue dans les milieux de vie des jeunes rend ces intervenants témoins des conditions de vie et des dynamiques qui influencent le parcours et les expériences des individus et des groupes qu'ils accompagnent. Cette première section explore les fondements de l'approche globale à partir de laquelle les acteurs en travail de rue appréhendent la problématique de l'intimidation en prenant en compte les déterminants sociaux de la santé, les rapports d'oppression et les ruptures sociales qui l'alimentent.

\subsection{L'impact des déterminants sociaux de la santé sur le phénomène de l'intimidation}

Depuis de nombreuses années, les protagonistes de l'action communautaire et plusieurs chercheurs en travail social insistent sur le fait que la prévention de toute problématique spécifique nécessite d'élargir le regard porté sur le phénomène ciblé de manière à avoir un impact réel et durable dans la vie des personnes concernées. À cet égard, dans un numéro de la revue Nouvelles Pratiques sociales (NPS) consacré à la mise en question de la logique de prévention précoce qui prédomine auprès des jeunes, Parazelli et ses collaborateurs (2012) déconstruisent les modèles biologistes réductionnistes sur lesquels s'appuie cette prévention, dont l'explication des troubles de conduite des enfants et des adolescents par leurs caractéristiques individuelles (et celles de leur entourage immédiat) tend à évacuer un ensemble " de facteurs sociaux, culturels, politiques, symboliques, idéologiques, etc. qui entrent en jeu dans ce type de réalités sociales complexes » (Parazelli, Lévesque et Gélinas, 2012, p. 3). Ces auteurs dénoncent le fait que la médicalisation des problèmes sociaux débouche sur des stratégies d'intervention axées presque exclusivement sur la modification des comportements individuels, incluant le recours à la médicamentation pour réguler ces derniers. Dans le même sens, Ridde (2004) estime que la focalisation des programmes publics sur la prévention des comportements à risques, au détriment d'une action plus englobante sur les déterminants sociaux de la santé, non seulement n'atténue pas les inégalités sociales à la source des problèmes sociaux mais de surcroît stigmatise les individus et les groupes qui vivent ces réalités.

Cette mise en perspective des enjeux soulevés par les modèles de prévention ciblée qui prédominent dans les champs social, sanitaire et sécuritaire (Parazelli, Lévesque et Gélinas, 2012) encourage à opérer un « pas de côté » par rapport à cette lecture symptomatique des problèmes sociaux afin de considérer l'influence des conditions de vie des personnes impliquées dans des situations d'intimidation. En effet, dès lors qu'on admet que l'intimidation, comme d'autres problèmes sociaux, prend racine et s'alimente dans les rapports sociaux inégaux (CDSS, 2009; Dorvil, 2013), on peut penser qu'un plan de lutte contre ce phénomène ne peut trouver son efficacité que s'il s'intègre dans une stratégie plus large de promotion de la santé, tel que définie par la Charte d'Ottawa comme un « processus qui confère aux populations les moyens d'assurer un plus grand contrôle sur leur propre santé et d'améliorer celle-ci » (OMS, 1986, p. 1). Dans la même optique, et suivant la définition de la santé formulée dans l'article 1 de la Loi sur la santé et les services sociaux du Québec en tant que « capacité physique, psychique et sociale des personnes d'agir dans leur milieu et d'accomplir les rôles qu'elles entendent assumer d'une manière acceptable pour elles-mêmes et pour les groupes dont elles font partie », on peut présumer que l'action sur les déterminants sociaux de la santé (revenu, éducation, emploi, 
logement, accès aux services, réseaux sociaux, reconnaissance sociale, participation citoyenne) représente une stratégie de prévention avalisée pour contrer un phénomène comme celui de l'intimidation. En somme, en parallèle aux campagnes de sensibilisation, la prévention de ce phénomène commande le déploiement de stratégies d'action orientées vers l'amélioration des « conditions dans lesquelles les personnes naissent, grandissent, vivent, travaillent et vieillissent » (CDSS, 2009; Gouvernement du Québec, 2012).

\subsection{Les facteurs d'oppression qui alimentent les dynamiques d'intimidation}

Comme l'accès aux ressources et au pouvoir n'est pas équitablement réparti dans la société, la prise en compte de l'influence des déterminants sociaux de la santé dans l'émergence des problèmes sociaux invite à poser une analyse structurelle de l'intimidation afin de mieux comprendre comment ce phénomène s'enracine dans les rapports sociaux d'oppression.

Plusieurs statistiques mettent en lumière le lien étroit entre le recours à l'intimidation au cours de l'enfance et l'adoption de comportements délinquants et antisociaux à l'adolescence et à l'âge adulte (CNCP, 2008; Day et Wanklyn, 2012). La recension d'écrits réalisée par le Centre national de prévention du crime (CNCP) note également que les facteurs de risques relatifs à l'intimidation sont sensiblement les mêmes que ceux reliés à la délinquance (absentéisme scolaire, comportements agressifs, manque de respect envers l'autorité, etc.) et que ces problèmes sont souvent associés, en particulier du côté des garçons. Si les facteurs individuels explicatifs de ces deux problèmes sociaux sont bien documentés dans les études recensées, il paraît pertinent aussi de chercher à comprendre les facteurs structurels qui poussent certains jeunes à recourir à une forme ou une autre de violence envers autrui (Devine, 2001; McMurtry et Curling, 2008).

Bien qu'on ne puisse établir un lien de causalité direct entre les facteurs structurels et le recours à la violence, McMurtry et Curling (2008) y associent plusieurs conditions sous-jacentes qui alimentent les facteurs de risque immédiat de recourir à la violence (pauvreté, racisme, manque de perspective d'avenir, etc.). Machado Azeredo et al. (2015) montrent quant à eux comment certains facteurs contextuels (inégalités sociales, manque d'accès à des services, manque de support des adultes, etc.) participent aux causes et aux conséquences de l'intimidation, alors que la qualité de vie dans un milieu conditionne le mode de vie et les opportunités dont y disposent les individus et ainsi influence leur état de santé global. La reconnaissance de ces facteurs contextuels invite à s'éloigner d'une explication strictement individualisante des comportements reliés à l'intimidation afin de considérer les facteurs sociaux qui peuvent ouvrir la voie à l'émergence de ce phénomène dans un milieu ou encore activer certaines caractéristiques individuelles qui accentuent les risques de perpétrer ou de subir de l'intimidation (Machado Azeredo et al., 2015).

La prise en compte des racines sociales, et non seulement individuelles, de l'intimidation engage une remise en question des rapports d'oppression auxquels s'alimentent de telles relations d'abus de pouvoir, au sein même de la culture ambiante (Devine, 2001; Krug et al., 2002; McMurtry et Curling, 2008). Dans cette perspective, Garnett et al. (2014) croient qu'il ne faut pas sous-estimer les liens entre les multiples formes de discrimination qui existent au sein de la société et la détresse émotionnelle que peuvent ressentir les jeunes qui y sont confrontés, 
alors que l'intimidation qu'ils vivent fait écho à des souffrances profondément ancrées dans des rapports sociaux aliénants. En outre, si le poids de l'oppression subie est relativement perceptible du côté des victimes, certains auteurs montrent comment les agresseurs sont souvent eux aussi piégés à l'intérieur de rapports sociaux oppressifs. McMurtry et Curling (2008) soulignent à quel point la discrimination, entre autres le racisme, participe directement au cercle vicieux de la violence chez les jeunes, en érigeant des blocages sociaux générateurs de frustrations. Comme l'élaborent Hintermeyer (2003), Castel (2007) et Mohammed (2014), l'usage de la violence par des jeunes délinquants est en même temps un symptôme de la violence structurelle subie dans un contexte sociétal défavorisant pour plusieurs. Aussi, comme le relève Derivois (2010), le recours à la violence s'inscrit parfois dans une quête d'adéquation envers ce qu'un jeune pense que l'environnement attend de lui.

\subsection{Les ruptures de liens sociaux comme causes et conséquences de l'intimidation}

Partant d'une telle analyse structurelle du phénomène de l'intimidation, on comprend que cette dynamique s'avère souvent la résultante d'une rupture sociale qui prend racine dans une souffrance familiale, identitaire, relationnelle, économique ou sociale (Paugam, 2014). En fait, bien que les tensions engendrées par une rupture sociale puissent mener à une violence envers soi-même, ces souffrances peuvent aussi pousser une personne à user de violence pour gagner du pouvoir sur autrui. En effet, comme l'explicite Derivois (2010), le cumul de petites et de grandes violences (dans la famille, parmi les pairs, au sein des institutions) crée des manques dans le fil de I'histoire des jeunes délinquants, cultivant au fur et à mesure un rapport d'attaque à leur environnement, comme en témoigne ce jeune pour qui faire subir de l'intimidation aux autres représente une façon d'éviter de la subir lui-même : "Si je veux que personne ne me tape dessus, il faut que cette personne [sic] ait peur de moi, c'est tout simple » (cité par Hintermeyer, 2003, p. 82). Or, comme l'abus de pouvoir peut s'actualiser dans une autre sphère de vie que celle d'où provient la souffrance ressentie, on peut difficilement identifier la cause directe d'un acte d'intimidation. Par exemple, comme le décrivent Trifan et Stattin (2015), les jeunes qui vivent des relations hostiles à la maison avec leurs parents sont ceux qui sont le plus à risque d'être impliqués dans des situations d'intimidation à l'école et dans les espaces qu'ils fréquentent dans leurs temps libres.

Considérer les ruptures sociales dont originent les dynamiques d'intimidation et qu'elles engendrent mène à concevoir qu'elles exposent non seulement les victimes à l'humiliation et à l'insécurité, mais que le phénomène prend lui-même racine et se nourrit de diverses formes de déficit de protection et de déni de reconnaissance (Paugam, 2014). Un tel point de vue suggère de considérer qu'un déficit de protection (pouvoir, ressources, statut, etc.) est non seulement perceptible dans le drame vécu par la victime, de plus en plus isolée dans une position fragilisée, mais aussi dans la stratégie défensive de celui qui agresse pour détourner l'offensive (Le Breton, 2003; Hintermeyer, 2003; Mohammed, 2014) ou dans l'accord tacite des témoins qui laissent aller cet engrenage de violence, de peur d'eux-mêmes précariser leur statut parmi les autres (McMurtry et Curling, 2008; Rivers et al., 2009). Une telle perspective invite aussi à reconnaître que, à la source du geste de l'agresseur, dans les conséquences sur l'intimidé et dans la retenue des témoins, peut être projeté, subi ou anticipé un déni de reconnaissance (rejet, étiquette, exclusion, relégation, discrimination, etc.). Le fait que les jeunes à la fois victimes et agresseurs 
soient ceux qui cumulent le plus de problèmes internalisés et externalisés (Rivers et al., 2009; Trifan et Stattin, 2015) renforce l'hypothèse d'une interaction entre l'humiliation subie et I'humiliation provoquée.

Parmi les ruptures sociales susceptibles de pousser les jeunes à rechercher des formes compensatoires de reconnaissance et de protection (Mohammed, 2014), plusieurs auteurs (Bondu, 1998; Parazelli, 2002; Le Breton, 2003) ont largement documenté comment le brouillage normatif ambiant et les difficultés d'intégration sociale incitent les jeunes à adopter des pratiques à risques, dont l'usage de la violence et l'intimidation constitue un exemple. Dans un contexte où les jeunes sont de plus en plus appelés à bricoler eux-mêmes leurs repères pour éprouver et prouver leur valeur (Parazelli, 2002; Jeffrey, Le Breton et Lévy, 2005), ceux qui ne disposent pas de ressources socialement valorisées ont plus tendance à adopter des conduites à risque pour donner sens à leur existence et faire leur place en société. Comme le souligne Le Breton, «à l'image des autres conduites, la violence est un jeu avec la limite, une manière de se heurter au monde à défaut d'une butée pour s'enraciner et trouver la distance propice au lien social » (2003, p. 35). II ajoute que la violence et l'intimidation sont souvent des stratégies adoptées par les jeunes pour se positionner dans les rapports de force avec autrui, faute d'être en mesure d'établir un rapport de sens dans ces relations. Ainsi, la présence des autres les amènerait à « vivre dans un défi permanent, à sans cesse vouloir montrer aux autres qu'on est à la hauteur » (Le Breton, 2003. p. 35). Ce sentiment de nécessité se renforce d'ailleurs au fur et à mesure que les jeunes qui adoptent ces comportements se reconnaissent dans l'identité délinquante construite au fil des interactions négatives qu'ils entretiennent avec les institutions (Carra, 2005; Castel, 2007; Derivois, 2010).

En somme, la prise en compte des facteurs sociaux sous-jacents à l'émergence et à la persévérance de dynamiques d'intimidation est nécessaire pour prévenir l'engrenage de la violence dans laquelle s'inscrit ce phénomène. Inversement, ne pas tenir compte de ces facteurs et ne s'attarder qu'aux comportements des jeunes intimidateurs risque d'amplifier la contribution des institutions à la construction de l'identité délinquante de ceux qui s'y adonnent, et donc à la reproduction de modes conflictuels de relation au monde qui les entoure (Carra, 2005; Derivois, 2010).

\section{Le travail de rue, une intervention de proximité au cœur des interactions quotidiennes}

Si la position des travailleurs de rue dans la vie quotidienne des personnes les amène à prendre en compte les facteurs structurels qui participent aux cycles de la violence, cette posture de proximité conditionne aussi l'approche globale d'intervention que privilégient ces intervenants auprès des personnes impliquées directement ou indirectement dans des dynamiques d'intimidation.

\subsection{Une présence favorable au maillage de liens sociaux dans la communauté}

L'implication de la communauté constitue un atout majeur dans les programmes de lutte contre l'intimidation (McMurtry et Curling, 2008; CNCP, 2008). Or, pour que les citoyens de la communauté puissent envisager faire une différence dans la vie des personnes concernées, il 
importe de penser leur rôle au-delà de la dénonciation des situations d'intimidation dont ils peuvent être témoins. Dans cette perspective, encourager la sociabilité au cœur de la communauté représente une stratégie incontournable pour favoriser le mieux-être de la collectivité et ainsi y faciliter l'aide mutuelle et la solidarité. En somme, le maillage du tissu social au sein de la communauté, tant au plan des liens informels que des liens formels, constitue une clé de la lutte contre l'intimidation, comme le confirment les auteurs d'un rapport portant sur l'examen des causes de la violence chez les jeunes en Ontario : « En donnant aux habitants la possibilité d'agir de manière positive pour transformer leurs quartiers en des lieux où il fait bon vivre, on crée un "cycle vertueux" où l'action communautaire réduit l'impact des causes de la violence à mesure que la communauté se responsabilise à l'échelon local » (McMurtry et Curling, 2008, p.145).

Par leur approche d'action communautaire et d'intervention de proximité, les travailleurs de rue sont des acteurs proactifs dans cet exercice de tissage de liens sociaux (Cheval, 2001; de Boevé et Giraldi, 2010; Fontaine, 2013a). La diversité des liens qu'ils entretiennent avec des individus et groupes sociaux variés (décideurs, intervenants, commerçants, résidents, jeunes du quartier) place les travailleurs de rue dans une position d'interface propice à tisser des passerelles entre des acteurs qui ont peu d'occasions d'entretenir des interactions positives. Cette position permet aussi aux travailleurs de rue de s'associer à divers alliés communautaires et institutionnels pour agir collectivement sur les déterminants sociaux de la santé qui affectent le bien-être des jeunes et de leur entourage : amélioration de l'adéquation et de l'accès des services sociaux, favorisation d'un climat inclusif dans la communauté, soutien au développement de lieux et de liens d'appartenance, promotion du droit des jeunes à prendre leur place dans l'espace public, etc.

\subsection{Un dialogue conscientisant favorable à des interactions constructives entre pairs}

Comme les dynamiques d'intimidation mettent souvent en scène des rapports de domination inscrits dans la culture ambiante (Garnett et al., 2014), contrer ce phénomène implique d'accompagner un processus de conscientisation des personnes à l'égard des postures d'opprimé et d'oppresseur auxquelles astreignent les divisions sociales discriminatoires (hétérosexisme, racisme, genrisme, capacitisme, etc.) (Freire, 2006; Pullen-Sansfaçon, 2013). À plus forte raison, lorsque l'intimidation prend forme parmi des groupes de jeunes eux-mêmes confrontés à diverses inégalités sociales, ne pas considérer l'oppression dont ils sont victimes constitue une violence structurelle à risque d'alimenter leur besoin de recourir à la violence pour gagner une reconnaissance et exister socialement (Castel, 2007).

La présence des travailleurs de rue dans les espaces que fréquentent les jeunes et la relation ouverte qu'ils établissent avec eux leur donnent l'occasion de s'approprier un questionnement personnalisé au sujet des enjeux de pouvoir et d'identité souvent prétextes à l'intimidation (Fontaine, 2013a). Ainsi, dialoguer de manière informelle de divers sujets, par exemple de l'orientation sexuelle, des rapports de genre ou des relations interculturelles, permet aux travailleurs de rue d'aborder des sujets tabous qui, lorsqu'ils restent occultés, donnent lieu à diverses situations d'intimidation. L'accessibilité de ces adultes, qui agissent comme catalyseur parmi les jeunes, qui prennent en considération leurs préoccupations, qui font écho à leurs 
attitudes, qui interrogent leurs gestes et leurs paroles, permet aux jeunes de réfléchir, in vivo, à leurs expériences et relations. En somme, l'ouverture au dialogue que permet la présence d'adultes accueillants dans les milieux de socialisation des jeunes crée des occasions d'expliciter et de démystifier les préjugés et ainsi de prévenir la dégradation des perceptions et des relations qu'ils entretiennent (Bondu, 1998; Martel, 2008; Fontaine, 2011).

En outre, à travers leurs interactions quotidiennes avec les jeunes, les travailleurs de rue sont à même de reconnaître leur esprit d'entraide. Holt et Espelage (2007) estiment que le soutien social entre pairs constitue une stratégie importante pour diminuer la détresse associée à l'expérience de la victimisation et pour encourager les intimidateurs à modifier leurs attitudes. À cet égard, les travailleurs de rue sont en bonne posture pour encourager les jeunes à tisser des liens d'aide mutuelle et à se solidariser face aux enjeux de l'intimidation. Ainsi, par leur influence positive parmi les jeunes, ces intervenants peuvent soutenir, comme le suggèrent Rivers et al. (2009), le développement de leur capacité à adopter une posture de défendeur (defender) plutôt que de témoin passif (outsider) ou de «renforceur » (reinforcer) en contexte d'intimidation, valorisant ainsi le potentiel des pairs à infléchir l'engrenage d'abus de pouvoir qui anime ce type de relation. En convergence avec les propos de Rivers et al. (2009), qui soulignent l'importance de développer les capacités empathiques chez les jeunes, van de Noorden et al. (2015) remarquent que les jeunes qui tendent à se positionner à la défense de leurs pairs intimidés sont ceux qui font le plus preuve d'empathie à la fois cognitive et affective. Comme le proposent ces auteurs, cette capacité que possèdent certains jeunes peut être un bon vecteur pour stimuler le développement de l'empathie auprès des autres jeunes. Ici encore, la proximité des travailleurs de rue avec les jeunes dans leurs interactions quotidiennes offre de multiples occasions de valoriser et de renforcer cette capacité ainsi que d'encourager l'influence positive des uns sur les autres.

\subsection{Un accompagnement personnalisé vers le mieux-être des jeunes concernés}

Ne diluant en rien la responsabilité de l'offense, le fait de porter un regard compréhensif, plutôt que seulement répressif, à l'égard d'un jeune qui intimide permet de repérer les aspects sur lesquels il est possible d'intervenir pour modifier la dynamique qui l'incite à recourir à la violence. Évitant de stigmatiser et de cristalliser leur identité délinquante (Carra, 2005), les travailleurs de rue adoptent une approche empathique et dialogique avec ces jeunes dont les comportements heurtent les normes sociales. En fait, grâce à leur attitude de non-jugement et au caractère volontaire des relations qu'ils établissent, les travailleurs de rue donnent l'occasion aux intimidateurs de poser un regard réfléchi et introspectif sur leur situation, sans crainte de se piéger à travers leurs révélations. Ces intervenants peuvent ainsi accompagner ces jeunes dans l'amélioration de leurs conditions d'existence en vue de diversifier la gamme d'options dont ils disposent pour réduire les tensions (économiques, sociales, psychologiques) qui les poussent à recourir à l'intimidation.

Dans un même esprit, les travailleurs de rue misent aussi sur l'établissement d'un rapport empathique et dialogique avec les jeunes intimidés en évitant de les réduire à leur statut de victime. Ainsi, comme c'est souvent l'anticipation des risques associés à la dénonciation qui entretient le silence (McMurtry et Curling, 2008) et fait reculer les personnes devant la déclaration 
des situations d'intimidation qu'elles subissent ou dont elles sont témoins (peur d'être perçu comme un faible, comme un délateur, d'empirer la situation, etc.), les travailleurs de rue prennent garde aux effets pervers d'une intervention qui renforcerait au lieu d'atténuer le sentiment de déni de reconnaissance et de déficit de protection ressenti en de telles circonstances (Paugam, 2014).

En somme, en valorisant les apprentissages qu'une telle situation permet de réaliser (conscience de soi et des autres, empathie, quête de justice sociale, affirmation, négociation, etc.) (Derivois, 2010), les travailleurs de rue cherchent à favoriser l'appropriation de pouvoir des personnes intimidées et ainsi les aident à faire face aux défis auxquels elles risquent à nouveau d'être confrontées, dans d'autres milieux, avec d'autres commettants.

\subsection{Une position pivot propice à la médiation des tensions et des relations}

La présence des travailleurs de rue dans la vie sociale de tous les jours place parfois ces intervenants de proximité en position d'exercer une médiation plus ou moins directe entre les acteurs impliqués dans des situations d'intimidation. Si leur proximité quotidienne leur permet de décoder et de dénouer certaines tensions, ou à tout le moins d'en prévenir les dommages, il arrive également qu'ils soient témoins de relations qui dérivent à petit feu ou qui éclatent en situations de crise. Dans la mesure où une relation de confiance est établie avec toutes les parties impliquées, ces intervenants peuvent jouer un rôle significatif dans la médiation de tels conflits.

Aussi, l'accompagnement individuel et collectif des personnes qui subissent, qui commettent ou qui sont témoins de gestes d'intimidation peut mener à un dénouement permettant de recréer des liens sociaux constructifs entre eux et avec d'autres. À cet égard, la possibilité d'offrir des lieux de socialisation pour les jeunes ainsi que des opportunités de projets qui les impliquent au sein de la communauté constitue un levier important pour favoriser une telle médiation des rapports sociaux (Bondu, 1998; Tétreault et Girard, 2007; de Boevé et Giraldi, 2010, Fontaine, 2011).

\section{La marge de manœuvre nécessaire pour déployer le plein potentiel de prévention et d'intervention du travail de rue en regard de l'intimidation}

Le présent article a mis en lumière la pertinence que des intervenants de proximité adoptent une lecture globale du phénomène de l'intimidation et déploient une pratique d'intervention généraliste. En guise de conclusion, cette dernière partie insiste sur les conditions nécessaires à entretenir pour assurer le déploiement du plein potentiel de cette pratique.

\subsection{Une posture de proximité qui exige ouverture, souplesse et non-jugement}

Être accepté dans les milieux de vie des jeunes, y gagner un statut crédible et s'y faire une place significative implique pour ces travailleurs de rue d'adopter une attitude d'ouverture, de souplesse et de non-jugement (Tétreault et Girard, 2007; Martel, 2008; Fontaine, 2010). Ainsi, pour que ces intervenants hors murs soient en mesure d'aller au plus près des personnes à risque de se retrouver dans des dynamiques d'intimidation, et pour qu'ils puissent établir une relation de confiance qui permette d'entrer dans un dialogue authentique, ils doivent pouvoir s'ouvrir au point de vue que les jeunes portent sur leur expérience et leurs enjeux, même dans les dimensions 
que ne souhaitent pas toujours entendre les adultes et les institutions sociales (Duval et Fontaine, 2000). Le respect dont font preuve les travailleurs de rue envers la dignité et l'intégrité des jeunes, même quand ceux-ci adoptent des comportements socialement inadéquats, et leur engagement à préserver le secret des confidences qu'ils reçoivent permettent de tisser une alliance émancipatrice avec ces jeunes.

Mettre à profit le travail de rue dans une stratégie concertée d'action contre l'intimidation signifie donc qu'il faut accorder à ces intervenants toute la marge de manœuvre dont ils ont besoin pour préserver les conditions de leur proximité avec les personnes en rupture sociale et ainsi assurer la portée de leur influence dans les situations rencontrées. Plus précisément, cela implique d'assumer que les travailleurs de rue ne se fassent pas les porte-voix des messages normatifs qui sont diffusés en regard de ce phénomène et qu'on évite de les enrôler dans les stratégies coercitives mises en œuvre pour contrer ce problème. Au contraire, ces intervenants doivent disposer d'un élastique souple et parfois intervenir dans l'ombre pour rejoindre les personnes qui se tiennent en marge des normes socialement admises (Fontaine, 2003).

\subsection{La nécessaire autonomie des organismes communautaires en travail de rue}

Dans cette perspective, pour que les travailleurs de rue apportent leur contribution singulière dans la panoplie d'interventions mobilisées pour réduire l'ampleur et les impacts de l'intimidation, il est essentiel de maintenir l'autonomie des organisations qui les embauchent, puisque l'efficacité des pratiques d'action communautaire repose d'abord sur l'adéquation de leur approche avec les besoins des personnes rejointes et sur l'ancrage de leurs initiatives dans les préoccupations et les aspirations des communautés où elles se déploient (Caillouette, 2001; Gouvernement du Québec, 2001; Bourque, 2003; Duval et al., 2005; Jetté, 2007; Fontaine, 2013b). Ainsi, alors que le confinement des acteurs communautaires dans un rôle programmé en fonction de priorités hétéro-déterminées compromet directement le potentiel et la portée de leurs actions, la valorisation de leur approche globale représente inversement une stratégie dont les bénéfices sur le bien-être de la communauté sont bien plus grands que les coûts engendrés (Lamoureux et al., 2012).

La mention du travail de rue dans le plan d'action gouvernemental de lutte contre l'intimidation récemment proposé par le gouvernement en place (Gouvernement du Québec, 2015a) laisse penser que la contribution d'une telle pratique d'intervention est désormais reconnue agir sur ce phénomène. Or, dans le contexte actuel de nouvelle gestion publique et d'austérité, guidé par la quête de résultats mesurables à court terme et au moindre coût, il est permis de se demander si la reconnaissance de l'utilité du travail de rue respectera les conditions de mise en œuvre nécessaires pour déployer le plein potentiel de cette pratique. Dans quelle mesure l'intérêt des autorités gouvernementales envers le travail de rue est-il centré sur l'opportunité d'utiliser cette pratique pour intervenir sur les comportements individuels des intimidateurs ? Dans quelle mesure l'éventuelle instrumentalisation du travail de rue à des fins d'intervention ciblée ne risquet-elle pas de tuer dans l'œuf cette pratique généraliste de promotion de la santé ?

Comme nous avons démontré dans cet article que l'efficacité de cette pratique repose en grande partie sur l'approche globale et l'attitude de non-jugement des travailleurs de rue, il est à 
espérer que la mise en valeur du travail de rue dans la lutte contre l'intimidation saura respecter ces conditions de façon à préserver la capacité de ces intervenants à briser le mur de méfiance qu'entretiennent plusieurs jeunes à l'égard des adultes et des institutions. En somme, c'est en misant sur leur capacité de manœuvrer dans les zones grises de la lutte contre l'intimidation, c'est-à-dire en marge des interventions plus conventionnelles, que les travailleurs de rue pourront le mieux accompagner les jeunes dans un processus d'amélioration de leurs conditions d'existence individuelles et collectives et ainsi contribuer à réduire l'incidence et les conséquences de ce problème social.

FONTAINE, Annie

PhD en service social Professeure adjointe à l'École de service social de l’Université Laval Membre de l'Observatoire Jeunes et Société

\section{RÉFÉRENCES}

Bondu, D. (1998). Nouvelles pratiques de médiation sociale. Jeunes en difficulté et travailleurs sociaux, Paris, ESF Éditeurs, coll. « Actions sociales / Société ».

Bourque, D. (2003). Essai de typologie du partenariat dans le secteur de la santé et des services sociaux au Québec, Gatineau, Université du Québec en Outaouais, Cahier du CÉRIS.

Bibeau, G., et M. Perreault (1995). Dérives montréalaises. À travers des itinéraires de toxicomanies dans le quartier Hochelaga-Maisonneuve, Montréal, Boréal, 234 p.

Caillouette, J. (2001). «Politiques de partenariat, pratique d'articulation identitaire et mouvement communautaire », Revue Nouvelles pratiques sociales, vol. 14, n 1, p. 81-96.

Carle, P. (dir.). (2014). Le travail de rue. Pratique " prometteuse » en matière de lutte à l'itinérance! Pratique "nécessaire » auprès des jeunes! Une recherche sur le travail de rue dans les Laurentides et à Laval, L'Écluse des Laurentides et le TRIL.

Carra, C. (2005). « Le délinquant comme produit de la dialectique identité personnelle / régulations sociales. L'éclairage de l'approche biographique » dans N. Brunelle et M.-M. Cousineau, Trajectoires de déviance juvénile. Les éclairages de la recherche qualitative, Québec, Presses de l'Université du Québec, p. 153-178.

Castel, R. (2007). La discrimination négative. Citoyens ou indigènes ?, Paris, Éditions du Seuil.

CNCP (Centre national de prévention du crime) (2008). Prévenir l'intimidation. La nature et la fréquence de l'intimidation au Canada, Gouvernement du Canada, Sécurité publique Canada.

Clément, M., L. Gélineau et A.-M. McKay (dir.) (2009). Proximités. Lien, accompagnement et soin, Québec, Presses de l'Université du Québec. 
Cheval, C. (2001). « Des travailleurs de rue créateurs de liens sociaux », in H. Dorvil et R. Mayer (dir.), Problèmes sociaux, tome II : Études de cas et interventions sociales, Sainte-Foy, Presses de l'Université du Québec, p. 362-385.

CDSS (Commission des déterminants sociaux de la santé) (2009). Combler le fossé en une génération. Instaurer l'équité en agissant sur les déterminants sociaux de la santé, Genève, Organisation mondiale de la santé.

Day, D.M., et S. G. Wanklyn (2012). Détermination et définition des principaux facteurs de risque du comportement antisocial et délinquant chez les enfants et les jeunes, Ottawa, Centre national de prévention du crime.

De Boevé, E., et M. Giraldi (dir.) (2010). Guide international sur la méthodologie du travail de rue, Paris, L'Harmattan, 165 p.

Derivois, D. (2010). Les adolescents victimes-délinquants. Observer, écouter, comprendre, accompagner, Bruxelles, De Boeck.

Devine, J. (2001). « Le marché scolaire de la violence » in É. Debarbieux et C. Blaya (dir.), Violence à l'école et politiques publiques, Issy-les-Moulineaux, ESF Éditeur.

Dorvil, H. (2013). « Travail social et déterminants de la santé », Intervention, nº 139, p. 75-78.

Duval, M., A. Fontaine, D. Fournier, S. Garon, J.F. René (2005). Les organismes communautaires au Québec. Pratiques et enjeux, Montréal, Gaëtan Morin Éditeur.

Duval, M., et A. Fontaine (2000). «Lorsque des pratiques différentes se heurtent : les relations des travailleurs de rue avec les autres intervenants », Nouvelles Pratiques sociales, vol. 13, n 1, p. 49-67.

Fontaine, A. et S. Richard. (2014). Au cœur du quotidien, la contribution de l'action communautaire en travail de rue dans la lutte contre l'intimidation, mémoire soumis dans le cadre de la consultation publique sur la lutte contre l'intimidation organisé par le ministère de la Famille et des Aînés, 12 p.

Fontaine, A. (2013a). « Le travail de rue : accompagner les jeunes au fil de leurs aléas existentiels et quotidiens », Lien social et Politiques, $\mathrm{n}^{\circ} 70$, numéro thématique « Accompagnement des jeunes en difficulté », p. 189-203.

Fontaine, A. (2013b). «La quête d'autonomie de l'action communautaire au Québec. Mission ou illusion collective ? », Le Sociographe, numéro hors-série 6, « Les paradoxes de l'autonomie », p. 205-219.

Fontaine, A. (2011). «Le travailleur de rue, passeur et médiateur dans la vie des jeunes », in M. Goyette, C. Bellot et A. Pontbriand (dir.), Les transitions à la vie adulte des jeunes en difficulté. Concepts, figures et pratiques, Québec, Presses de l'Université du Québec, coll. « Problèmes sociaux et interventions sociales », p. 187-200.

Fontaine, A. (2010). « Le travail de rue dans l'univers de la rue », Criminologie, vol. 43, $n^{\circ}$ 1, dossier « Les jeunes et la rue », p. 137-153. 
Fontaine, A. (2003). « Aux marges du social, le travail de rue », Intervention, nº 119, p. 15-23.

Freire, P. (2006). Pédagogie de l'autonomie. Savoirs nécessaires à la pratique éducative, Ramonville-Saint-Agne, Ères.

Garnett, B.R., K.E. Masyn, S.B. Austin, M. Miller, D.R. Williams, K. Viswanath (2014). « The Intersectionality of Discrimination Attributes and Bullying Among Youth: An Applied Latent Class Analysis », Journal of Youth and Adolescence, vol. 43, nº 8, p. 1225-1239.

Gouvernement du Québec (2015a). Ensemble contre l'intimidation. Une responsabilité partagée. Plan d'action concerté pour prévenir et contrer l'intimidation 2015-2018, [Québec], ministère de la Famille.

Gouvernement du Québec (2012). La santé et ses déterminants. Mieux comprendre pour mieux agir, [Québec], ministère de la Santé et des Services sociaux.

Gouvernement du Québec (2015b). Loi sur la santé et les services sociaux, [Québec], ministère de la Santé et des Services sociaux.

Gouvernement du Québec (2001). Politique gouvernementale. L'action communautaire, une contribution essentielle à l'exercice de la citoyenneté et au développement social du Québec, [Québec], ministère de l'Emploi et de la Solidarité sociale.

Hintermeyer, P. (2003). « Les mots et les coups », in David Le Breton (dir.), L'adolescence à risque, Paris, Autrement, p. 67-89.

Holt, M.K., et D.L. Espelage (2007). «Perceived Social Support among Bullies, Victims, and BullyVictims », Journal of Youth and Adolescence, vol. 36, nº 8, p. 984-994.

Jetté, C. (2007). Les organismes communautaires et la transformation de l'État-providence. Trois décennies de coconstruction des politiques publiques dans le domaine de la santé et des services sociaux, Québec, Presses de l'Université du Québec, Coll. Pratiques et politiques sociales et économiques.

Jeffrey, D., D. Le Breton et J. J. Lévy (dir.) (2005). Jeunesse à risque. Rite et passage, Québec, Presses de l'Université Laval.

Krug, E.G., L.L. Dahlberg, J.A. Mercy, A. Zwi et R. Lozano-Ascencio (2002). Rapport mondial sur la violence et la santé, Genève, Organisation mondiale de la santé.

Lamoureux, H., A. Fontaine, M. Parazelli, F. Labbé, Relais-Femmes, A. Gauvin, S. Dusablon, M. Beaulieu (2012). L'approche globale. Contexte et enjeux. Réflexions d'un collectif d'auteurs, Québec, Regroupement des organismes communautaires de la région 03 (ROC 03)

Le Breton, D. (dir.) (2003). L'adolescence à risque, Paris, Autrement.

Machado Azeredo, C., A.E.M. Rinaldi, C. Leite de Moraes, R. Bertazzi Levy, P. Rossi Menezes (2015). «School bullying: A systematic review of contextual-level risk factors in observational studies », Aggression and Violent Behavior, vol. 22, p. 65-76. 
Martel, G. (2008). Le travail de rue : une pratique préventive auprès des jeunes à risque d'adhérer à un gang ?, Montréal, Société de criminologie du Québec pour la Direction de la prévention et de la lutte contre la criminalité, ministère de la Sécurité publique, 184 p.

McMurtry, R., et A. Curling (2008). Examen des causes de la violence chez les jeunes en Ontario, vol. 1 : Résultats, analyse et conclusions, Toronto, Imprimeur de la Reine pour l'Ontario.

Mohammed, M. (2014). «La force paradoxale des bandes de jeunes. Compensation et marginalisation sociales », in S. Paugam (dir.) L'intégration inégale. Force, fragilité et rupture des liens sociaux, Paris, Presses universitaires de France, p. 207-226.

OMS (Organisation mondiale de la santé) (1986). Charte d'Ottawa pour la promotion de la santé, 5 p.

Parazelli, M. (2002). La rue attractive. Parcours et pratiques identitaires des jeunes de la rue. Québec, Presses de l'Université du Québec, 358 p.

Parazelli, M., S. Lévesque et C. Gélinas (2012). « La prévention précoce en question », Nouvelles Pratiques sociales, hors-série $\mathrm{n}^{\circ} 1$, p. 1-15.

Paugam, S. (dir.) (2014). L'intégration inégale. Force, fragilité et rupture des liens sociaux, Paris, Presses universitaires de France.

Pullen-Sansfaçon, A. (2013). « La pratique anti-oppressive », in E. Harper et H. Dorvil (dir.) Le travail social. Théories, méthodologies et pratiques, Québec, Presses de l'Université du Québec.

Ridde, V. (2004). « Une analyse comparative entre le Canada, le Québec et la France : l'importance des rapports sociaux et politiques eu égard aux déterminants et aux inégalités de la santé », Recherches sociographiques, vol. 45, $\mathrm{n}^{\circ} 2$, p. 343-364.

Rivers, I., V.P. Poteat, N. Noret, N. Ashurst (2009). « Observing Bullying at School: The Mental Health Implications of Witness Status », School Psychology Quarterly, vol. 24, n 4, 211-223.

Tétreault, K., et G. Girard (2007). Rapport d'évaluation. Travail de rue, gang de rue, un lien incontournable?, Montréal, Société de criminologie du Québec pour le ministère de la Sécurité publique.

Trifan, T.A., et H. Stattin (2015). «Are Adolescents' Mutually Hostile Interactions at Home Reproduced in Other Everyday Life Contexts? », Journal of Youth and Adolescence, vol. 44, $\mathrm{n}^{\circ} 3$, p. 598-615.

Van de Noorden, T.H.J., G.J.T. Haselager, A.H.N. Cillessen, W.M. Bukowski (2015). "Empathy and Involvement in Bullying in Children and Adolescents: A Systematic Review", Journal of Youth and Adolescence, vol. 44, $\mathrm{n}^{\circ}$ 3, p. 637-657.

Wilkinson, R. (2004). «Why is violence more common where inequality is greater? », Annals of the New York Academy of Sciences, vol. 1036, $\mathrm{n}^{\circ}$ 1, p. 1-12. 